\title{
Perfil sociodemográfico y académico en estudiantes universitarios respecto a su autoeficacia académica percibida
}

\author{
Profile socio-demographic and academic in university students \\ regardingtheir perceived academic self-efficacy
}

ISSN 0124-0137

\author{
Luis Fernando Hernández Jácquez \\ Universidad Pedagógica de Durango, Victoria de Durango, México
}

Artículo de Investigación

Copyright 2018 by Psicogente

\section{Correspondencia de autores:}

Ifhj1@hotmail.com

Recibido: $26-09-16$

Aceptado: $18-07-17$

Publicado: 01-01-18

\section{Resumen}

Objetivo: Identificar el nivel de autoeficacia académica percibida en los estudiantes de una institución de educación superior del Estado de Durango, México y determinar el perfil sociodemográfico y académico de los estudiantes, en relación a su autoeficacia académica percibida

Método:El estudio se desarrolló bajo el enfoque cuantitativo de investigación, de diseño no experimental y transeccional, participando el total de la población estudiantil que cursa planes de estudio bajo el modelo de competencias profesionales, siendo 182 estudiantes, 46 $\%$ hombres y $54 \%$ mujeres, todos en edades de los 21 a los 23 años. El instrumento aplicado consistió en un cuestionario con escalamiento Likert $(\alpha=.91)$ más un apartado con información social, académica y demográfica

Resultados: De manera global, la autoeficacia académica percibida se ubica en un nivel medio; y variables como la seguridad de contar con algún tipo de beca, el gusto por la carrera que se estudia, el no tener dificultades económicas, el deseo por estudiar algún posgrado, la cantidad de asignaturas no aprobadas en el semestre antecesor, el promedio de bachillerato que se haya obtenido y el promedio del semestre anterior son variables que inciden directamente en la autoeficacia académica percibida

Conclusiones: El perfil socioacadémico definido, proporciona información útil que puede ser utilizada como diagnóstico de poblaciones con características similares a la que se estudió, y con ello, la institución podrá diseñar políticas y estrategias tendientes al aumento de su autoeficacia, y por ende al mejoramiento de su desempeño académico

Palabras Claves:

Autoeficacia académica, Educación superior, Perfil sociodemográfico, Perfil académico

\section{Abstract}

Objective: To identify the level of perceived academic self-efficacy in students of an institution of higher education in the State of DurangoMexico and to determine the sociodemographic and academic profile of the students, in relation to their perceived academic self-efficacy

Method: The study was developed under the quantitative approach of research, of non-experimental and transeccional design, involving the total of the student population that study curricula under the model of professional competences, being 182 students, $46 \%$ men and $54 \%$ women, and all aged 21 to 23 years. The instrument used consisted of a questionnaire with Likert scaling $(\alpha=.91)$ plus a section with social, academic and demographic information

Results: Overall, perceived academic self-efficacy is at an intermediate level; and variables such as the security of having some kind of scholarship, the pleased by the university career, not having economic difficulties, the desire to study a postgraduate course, the amount of subjects not approved in the previous semester, the average of high school and the average of the semester previous are variables that affect directly in the perceived academic self-efficacy

Conclusion: The defined socio-academic profile provides useful information that can be used as a diagnosis of populations with characteristics similar to the one studied, and with this, the institution can design policies and strategies tending to increase its selfefficacy, and therefore improvement of academic performance.

Key words:Academic self-efficacy, Higher education, Sociodemographic profile, Academic profile

Como citar este artículo (APA):

Hernández, L. (2018). Perfil sociodemográfico y académico en estudiantes universitarios respecto a su autoeficacia académica percibida. Psicogente, 21(39), 35-49. http://doi.org/10.17081/psico.21.39.2820 


\section{INTRODUCCIÓN}

En el presente apartado se describe una serie de investigaciones que dan cuenta de la importancia del estudio de la autoeficacia. Se presenta el planteamiento del problema que dio origen a la presente investigación, y sus preguntas y objetivos, así como el fundamento teórico del estudio.

\subsection{Antecedentes}

La autoeficacia académica es un constructo que en años recientes ha tenido una evolución importante en cuanto a investigación empírica se refiere, específicamente está siendo muy estudiada en el ámbito médico, deportivo y escolar.

De manera general y atendiendo al ámbito escolar, en los últimos años se ha relacionado a la autoeficacia con una serie de variables, tal como en el estudio de García, Inglés, Vicent, Gonzálvez, Lagos y Pérez (2016), quienes analizaron la capacidad predictiva de la autoeficacia académica percibida sobre las altas autoatribuciones académicas en estudiantes adolescentes chilenos, encontrando una predicción positiva en situaciones de éxito y negativa ante situaciones de fracaso.

Con el objetivo de analizar la relación entre las estrategias de afrontamiento ante la situación preexamen y la autoeficacia académica en una muestra de estudiantes de ciencias de la salud, Domínguez (2016) llevó a cabo un estudio con 208 estudiantes entre 16 y 35 años, encontrando que las personas con mayor autoeficacia académica tienden a usar estrategias más vinculadas con el control de la situación previa al examen, que las destinadas a evitar o buscar apoyo.

En un aspecto distinto, Kyle, Hernández, Reigal y Morales (2016) evaluaron los efectos de la práctica física regular sobre el autoconcepto y la autoeficacia en un grupo de preadolescentes, a través de un estudio cuasi experimental, encontrando efectos significativos de dicha práctica física sobre el autoconcepto físico y el autoconcepto académico, además de indicios de significación en la autoeficacia académica y la autoeficacia social.

Por su parte, García, Díaz, Torregrosa, Inglés, Lagos y Gonzálvez (2016) determinaron la capacidad predictiva de la autoeficacia académica sobre las dimensiones del autoconcepto en estudiantes chilenos, indicando que dicha autoeficacia fue un predictor positivo y significativo de las escalas académicas matemáticas, verbal y académica general; y de las escalas no académicas como las habilidades físicas, apariencia física, relaciones sociales con personas del mismo sexo y del sexo opuesto; y de la escala de autoestima.

Hernández (2016) estudió la relación entre las variables autoeficacia académica y estrés académico en estudiantes de licenciatura en México, concluyendo que estadísticamente no fue posible establecer una correlación significativa entre ambas variables.

Además de la muestra de los antecedentes descritos anteriormente, la autoeficacia ha sido estudiada por Gómez, Villegas, Barrea y Cruz (2007) en relación con el bienestar subjetivo; porMedrano (2011) en relación al modelo social cognitivo del rendimiento académico, y por Alpuche y Vega (2014) para predecir el comportamiento lector en alumnos de primer grado de primaria; así como por Aluicio y Revellino (2011), quienes revelaron que, contrariamente a lo 
esperado, la autoeficacia no mostró una correlación significativa con el rendimiento académico, en una muestra de estudiantes chilenos de la Universidad San Sebastián.

Descrita como factor para el mejoramiento de las actitudes y de la motivación, la autoeficacia ha sido abordada por Barraza, Martínez, Silva, Camargo y Antuna (2012); Sánchez (2013); Ornelas, Blanco, Gastélum y Chávez (2012); Alves (2013); Camposeco (2012); Piemontesi y Heredia (2011); yVeliz y Apodaca (2012).

Por último, como ejemplos del estudio de la relación de la autoeficacia académica con variables sociodemográficas y económicas, se tienen las investigaciones de Blanco, Ornelas, Aguirre y Gueda (2012); Rosado (2013); Catzin, Esquivel y Canto (2011); Rosário (2012); Galicia, Sánchez y Robles (2013); y Méndez, Peña e Inda (2012).

Para un panorama mucho más preciso del estado del conocimiento de la autoeficacia académica, se sugiere al lector consultar el realizado por Hernández (2016).

\subsection{Planteamiento del problema}

La presente investigación parte de la inquietud de poder establecer las características sociodemográficas y académicas de los estudiantes de una institución de educación superior en el estado de Durango, México, relacionadas con el nivel de autoeficacia percibida por ellos mismos.

Esta inquietud nace de la experiencia propia de los profesores y el cuerpo de tutores de la institución, quienes han notado disparidad en el rendimiento académico de los estudiantes tanto pertenecientes a un mismo grupo, como de manera intergrupal.

Si bien, por sí mismo lo anterior no representa algo extraordinario en las instituciones educativas en México, se ha comenzado a asociar esta diferenciación académica a la percepción que cada estudiante tiene de su capacidad para llevar a cabo las labores escolares, lo que se ha detectado con los distintos mecanismos y herramientas a través del Programa Institucional de Tutorías.

De acuerdo a los antecedentes revisados, es claro que la relación de la autoeficacia con diversas variables le ha permitido considerarse como un elemento predictor de acciones futuras de las personas en distintos aspectos del ámbito escolar o académico, tal como lo demuestran los resultados de García et al. (2016), Domínguez (2016), García et al. (2016), Medrano (2011); y es esta justamente la finalidad última de esta investigación: el poder emplear las características de los estudiantes en relación a la autoeficacia académica como predictor de su posible éxito académico (Alpuche \& Vega, 2014).

Así, el contexto en que se ubica la institución, le permite tener estudiantes de muy diversas condiciones sociales y demográficas, siendo que recibe alumnado tanto del medio rural y semiurbano, como del urbano. En cuanto a su matrícula, se alimenta de al menos seis escuelas de educación media superior, lo que incluye el currículum de bachillerato general, bachillerato tecnológico agropecuario, bachillerato forestal y bachillerato tecnológico industrial. Las edades de los estudiantes se encuentran en un rango amplio, ya que se reciben tanto estudiantes recién 


\section{Luis Fernando Hernández Jácquez}

egresados del nivel medio superior como estudiantes rezagados de acuerdo a la edad escolar promedio, que se atienden en grupos especiales a contra turno.

Parte de este conjunto de características se complementan con quienes estudian y a la vez están integrados al medio laboral, hay quienes viven con sus padres y quienes viven con personas distintas a ellos, o quienes viven solos. Existen estudiantes que si logran culminar su carrera serán los primeros profesionistas de su familia, y en fin, las características poblacionales son bastante variadas.

Los antecedentes muestran también, que la autoeficacia se ha estudiado en relación con diversas variables sociales, demográficas y económicas (Blanco et al., 2012; Rosado, 2013; Catzin et al, 2011; Rosário, 2012; Galicia et al., 2013; Méndez et al., 2012); pero en ningún caso, se ha establecido (por los propios objetivos de los estudios) un perfil completo que pueda describir las características de los sujetos ante tales o cuales situaciones, por lo que se considera importante identificar aquellas características que están asociadas a la percepción de autoeficacia académica de los sujetos de estudio, para poder predecir hasta donde sea posible el esfuerzo que emplearán los estudiantes en sus estudios. Además, con dichas características identificadas se podrían establecer estrategias para el mejoramiento académico de quienes presentan bajos niveles, siempre y cuando esto se encuentre relacionado con la autoeficacia académica percibida.

Por lo tanto, la investigación plantea las siguientes preguntas generales:

1. ¿Cuál es el nivel de autoeficacia académica percibida en los estudiantes de una institución de educación superior del Estado de Durango, México?

2. ¿Cuál es el perfil sociodemográfico y académico de los estudiantes que guarda relación con su autoeficacia académica percibida?

En correspondencia a las preguntas de investigación, los objetivos de la misma son:

1. Identificar el nivel de autoeficacia académica percibida en los estudiantes de una institución de educación superior del Estado de Durango, México.

2. Determinar el perfil sociodemográfico y académico de los estudiantes, en relación a su autoeficacia académica percibida.

\subsection{Teoría base}

Teoría de la Autoeficacia de Bandura Aunque la teorización de la autoeficacia se originó de la teoría del aprendizaje social de Rotter, fue Albert Bandura en 1977 quien la desarrolló de manera amplia a través de su teoría sociocognitiva o cognitiva social. De acuerdo con Peralbo, Sánchez y Simón (1986) el concepto de autoeficacia surge como un intento de conceptualizar la mayor parte del comportamiento humano desde una perspectiva integradora. Esta perspectiva implicaba necesariamente el recurso a una explicación de los determinantes de la conducta humana que toma su origen en posturas centralistas derivadas del auge de la psicología cognitiva, en clara oposición a las posturas periferialistas que sitúan los determinantes de la conducta en el ambiente externo. 
En el contexto del estudio y la medida de la personalidad, la autoeficacia se podría inscribir en el marco de las distintas concepciones del self (Palenzuela, 1983, citado por Peralbo et al., 1986), que dentro de un enfoque interaccionista de la personalidad se llega a la consideración de las variables de la persona no como los clásicos rasgos globales que describen su conducta sino más bien como las cogniciones acerca de las situaciones concretas. Blanco (2010, p.2) asienta que de manera general, de la Teoría Cognitiva Social se desprenden dos preposiciones:

a) Las creencias de autoeficacia son específicas de un ámbito de funcionamiento dado

b) el constructo de autoeficacia puede ser distinguido de otros constructos autorreferentes cuando se evalúa en relación con un dominio específico.

Las creencias de la autoeficacia se refieren a los juicios que cada individuo hace acerca de sus capacidades para llevar a cabo una tarea. Bandura (1997, citado por Chacón, 2006, p.2) plantea que los individuos poseen un sistema interno que les permite ejercer control sobre sus propias acciones, conductas y pensamientos, siendo este sistema un componente fundamental de influencia en el logro de las metas que cada quien se propone. El mismo Bandura (1997, citado por Chacón, 2006, p.2) señala que "las creencias de autoeficacia constituyen un factor decisivo en el logro de metas y tareas de un individuo. Si las personas creen que no tienen poder para producir resultados, no harán el intento para hacer que esto suceda".

Parafraseando a Bandura (1977), la percepción de autoeficacia se define como el conjunto de juicios de cada individuo sobre sus propias capacidades para organizar y ejecutar las acciones o tareas necesarias en el manejo de posibles situaciones específicas. Estos juicios tienen importantes efectos sobre la elección de conductas o actividades, sobre el esfuerzo empleado y la persistencia, y sobre los patrones de pensamiento y las reacciones emocionales ante las tareas.

En general, el control y la competencia personal que los individuos poseen como agentes creadores de su propio entorno no solo les permiten responder a su ambiente, sino que los capacita para transformarlo mediante su actuación o desempeño proporcionando a la persona un mecanismo de referencia a partir del cual se percibe y evalúa el comportamiento humano. De ello se desprende que si el sujeto se juzga capaz y confía en sus habilidades de ejecución de una tarea específica, esta autopercepción contribuirá al éxito de su desempeño, por lo tanto podría decirse que el éxito esperado en el logro de una meta se relaciona de manera directa con las expectativas de resultado que el sujeto anticipa.

Este sistema interno de creencias proporciona un marco referencial determinante de la percepción, regulación y evaluación de la conducta del individuo. De allí la importancia de considerar las creencias de autoeficacia como el juicio personal sobre las capacidades o autoconfianza en la ejecución de tareas propuestas en el momento de llevar a cabo lo que se intenta hacer. Las preconcepciones o juicios que la persona establece acerca del éxito o fracaso de su desempeño proporcionan información que a su vez, alteran las creencias de autoeficacia percibida que se reflejarán en desempeños posteriores. 
Siguiendo a Bandura (1977, citado por Blanco, 2010, p.2) la autoeficacia no es un rasgo global sino un conjunto de autocreencias ligadas a ámbitos de desempeño diferenciados. La teoría mantiene que los sujetos no solo emiten juicios de autoeficacia diferenciados y diferenciables para dominios diversos, sino que la autoeficacia puede y debe definirse con distintos niveles de especificidad en relación con un ámbito dado, de acuerdo con la conducta y criterio de interés.

Así, los individuos evalúan sus propias experiencias mediante la autorreflexión, de manera que el juicio o creencias personales sobre las capacidades y logros previos ejercen una fuerte influencia en las actuaciones futuras. De esta manera, las creencias de autoeficacia pueden anticipar mejor el comportamiento futuro del sujeto que la actuación previa, sin embargo, es importante aclarar que en la fundamentación de esta teoría las tareas que una persona es capaz de ejecutar no pueden ir más allá de sus capacidades, ya que no basta solo con creer en las capacidades que se poseen, sino que se requiere de las propias habilidades y conocimientos necesarios para el logro de una competencia adecuada.

\subsection{Fuentes de autoeficacia}

Los individuos adquieren retroalimentación acerca de su autoeficacia en determinada área o tarea a partir de sus propias ejecuciones (experiencias directas, de dominio), de las observaciones de modelos (experiencias vicarias), de formas de persuasión social (verbal, simbólica) y de índices (activación, estado) fisiológicos (Schunk, 2012).

Los elementos anteriores son conocidos como las fuentes de la autoeficacia y se conceptualizan como sigue:

\subsubsection{Experiencias directas (de dominio).}

La fuente más influyente de información sobre las capacidades de desempeño son los logros reales de desempeño o las experiencias personales de dominio. El éxito enseña a la gente que puede tener éxito... Más aún, cuando la base para el sentido de autoeficacia de una persona son sus propias acciones y logros, dicha base tiende a generalizarse a un rango de actividades similares (Bandura, 1997, citado por Tuckman \& Monetti, 2011).

\subsubsection{Experiencias vicarias, modelling o aprendizaje por observación.}

Ver que otros individuos similares desempeñan actividades con éxito puede elevar las percepciones sobre el nivel propio de autoeficacia (Schunk \& Miller, 2002, citados por Tuckman \& Monetti, 2011).

\subsubsection{Persuasión verbal (simbólica).}

Con esta fuente de autoeficacia, lo que se logra es convencer a la gente de que cuenta con la capacidad de hacer el trabajo si invierte suficiente esfuerzo (Bandura, 1997, citado por Tuckman \& Monetti, 2011). Básicamente "la eficacia de la persuasión verbal depende de la credibilidad percibida y de la experiencia de quien persuade" (Tuckman \& Monetti, 2011, p.396). 


\section{Luis Fernando Hernández Jácquez}

\subsubsection{Activación (estado) fisiológica.}

Los estados emocionales de una persona ejercen influencia sobre el sentido de eficacia desde la perspectiva de que la activación fisiológica evidenciada en ansiedad, estrés, estado de ánimo, y temores, afectan el desempeño del individuo. "La gente depende de su estado emocional o estado de activación para juzgar si es capaz o no de hacer algo con éxito" (Tuckman \& Monetti, 2011, p.396).

\section{MÉTODO}

\subsection{Diseño}

La investigación se desarrolla bajo el enfoque cuantitativo, de manera que se recolectan datos con la finalidad de probar estadísticamente las hipótesis planteadas (Hernández, Fernández \& Baptista, 2014) y descritas enseguida.

El alcance del estudio es correlacional, ya que "describe la relación lineal entre dos o más variables sin tratar en lo absoluto de atribuir a una variable el efecto observado en otra" (Salkind, 1999, p.223), de tipo no experimental transeccional, ya que no existe manipulación de las variables y la recopilación de datos se realizó en un solo momento.

Las hipótesis generales para el estudio son las siguientes:

H1: El nivel de autoeficacia académica percibida en los estudiantes de una institución de educación superior del Estado de Durango, es medio.

H2: Es posible determinar un perfil sociodemográfico y académico de los estudiantes en cuestión, descrito a partir de su autoeficacia académica percibida. Su contraparte, las hipótesis nulas quedan establecidas como sigue:

H01: El nivel de autoeficacia académica percibida en los estudiantes de una institución de educación superior del Estado de Durango, no es medio.

H02: No es posible determinar un perfil sociodemográfico y académico de los estudiantes en cuestión, descrito a partir de su autoeficacia académica percibida. Como se ha hecho saber, la variable central (principal) es la autoeficacia percibida, cuyas definiciones conceptual y operacional se muestran en la Tabla 1.

Tabla 1.

Definiciones conceptual y operacional de la variable autoeficacia percibida.

\begin{tabular}{|c|c|}
\hline Definición conceptual & Definición operacional \\
\hline $\begin{array}{l}\text { "Conjunto de juicios de cada individuo sobre las } \\
\text { capacidades propias para organizar y ejecutar las acciones } \\
\text { requeridas en el manejo de posibles situaciones } \\
\text { específicas" Bandura }(1977, \text { p. 80) }\end{array}$ & $\begin{array}{l}\text { Inventario de Expectativas de Autoeficacia Académica } \\
\text { (Barraza, 2010) }\end{array}$ \\
\hline
\end{tabular}




\subsection{Participantes}

Los participantes de la investigación representan todos aquellos estudiantes de la institución que cursan planes de estudio bajo el modelo de competencias profesionales. Se realizó un censo dada la facilidad y economía para la administración del instrumento de recolección. En total son 182 estudiantes, quienes constituyen los sujetos de investigación, que presentan, entre otras, las siguientes características:

a) Porcentaje de hombres y mujeres: $46,2 \%$ y $53,8 \%$, respectivamente.

b) Estudiantes del primero, tercero y quinto semestres: $33,4 \%, 47,3 \%$ y $19,3 \%$, respectivamente.

c) Rango de edad representativo de los participantes: 21 a 23 años (78,4 \% de los participantes).

d) Estudiantes becados: 58,7\%.

\subsection{Instrumentos}

La técnica para la recolección de datos es la encuesta y el instrumento un cuestionario conformado en dos apartados. En el primero se hace referencia al contexto sociodemográfico y académico del encuestado, y en el segundo apartado se utilizará el Inventario de Expectativas de Autoeficacia Académica (IEAA), con la finalidad de medir en lo particular la variable autoeficacia académica percibida; inventario validado en el Estado de Durango para estudiantes de educación media superior y superior por Barraza (2010).

Para el contexto sociodemográfico y académico, se construyeron un total de 17 ítems que indagaron acerca de las características de los estudiantes, a saber: género, estado civil, edad, si vive con sus padres en la localidad en donde se ubica la institución, si trabaja a la vez que estudia, si en su familia existen profesionistas; si existen dificultades económicas para mantenerse en sus estudios; si cuenta con algún tipo de beca, si la carrera que estudia es de su agrado, y si una vez que concluya la licenciatura ha pensado en estudiar algún posgrado. Sobre el contexto de procedencia del estudiante se preguntó acerca del tipo de institución de procedencia, el tipo de bachillerato y el tipo de comunidad de procedencia.

Para construir el perfil académico se preguntó sobre el promedio del semestre anterior (para los estudiantes del segundo semestre y posteriores), el número de asignaturas no acreditadas en el semestre anterior (también para estudiantes a partir del segundo semestre), el promedio de bachillerato y el puntaje obtenido en el examen de ingreso.

Por su parte, el Inventario de Expectativas de Autoeficacia Académica consta de 20 ítems que pueden ser respondidos en un escalamiento tipo Likert: nada seguro, poco seguro, seguro y muy seguro; y que da la pauta para responder a la actividad académica especificada en cada ítem bajo la premisa "¿qué tan seguro está de poder...?" El inventario muestra dentro de sus propiedades psicométricas un nivel de confiabilidad de .91 en alfa de Cronbach, y de .88 en la confiabilidad por 


\section{Luis Fernando Hernández Jácquez}

mitades. En una escala de 0 a 3, los ítems lograron una escala de 1,5 en cuanto a validez de contenido. La direccionalidad del instrumento es única, ya que así se comprobó a través del análisis de grupos contrastados, bajo un nivel de significación de .00 (Barraza, 2010).

Este inventario se divide en tres dimensiones: actividades académicas orientadas a la producción (output), actividades académicas de insumo para el aprendizaje (input), y actividades académicas de interacción para el aprendizaje (retroalimentación).

\subsection{Procedimiento}

Una vez definido el problema de investigación descrito anteriormente, se procedió a la aplicación del instrumento, para lo que se contó con la anuencia de las autoridades correspondientes. La aplicación tuvo una duración de cuatro días, en donde el aplicador (investigador) se hizo acompañar de la responsable del Programa Institucional de Tutorías, en horarios establecidos por ella. En cada aula y en el caso de los estudiantes que por algún motivo no estaban presentes durante la aplicación, y que posteriormente se les encuestó de manera individual, fue explicado el motivo del estudio y la finalidad del cuestionario, con el objetivo de que se contestase de la manera más veraz posible. Una vez que los estudiantes respondían el instrumento, en ese momento se realizaba una inspección visual para asegurarse de que en todos los ítems hubiese respuesta. No se presentaron incidentes en ningún momento y al final de la investigación, los resultados se hicieron llegar a la institución.

\subsection{Análisis de datos}

La información recabada se procesó mediante el software IBM SPSS Statistics 20. Para dar respuesta a las preguntas de investigación se utilizó estadística descriptiva e inferencial, empleando la prueba t de Student, ANOVA y la correlación de Pearson, según la naturaleza de los datos lo demandase.

\subsection{Conflicto de interés}

En todo el proceso de investigación y hasta la entrega de los resultados a la institución, no se tuvo ninguna situación de conflicto de interés real o potencial, incluyendo ningún interés personal, de autoridades de la institución, o financiero.

\section{RESULTADOS}

El resultado general para la autoeficacia académica es 1,89 puntos (en escala de 0 a 3), que de acuerdo al baremo propuesto por Barraza (2010), equivale a 63,0 \%, lo que significa un nivel medio de autoeficacia académica percibida.

El análisis dimensional arroja que para la dimensión output, la media aritmética es 1,93 puntos, que convirtiéndolo en porcentaje equivale a $64,33 \%$, lo que significa que los estudiantes presentan un nivel medio de confianza para poder realizar exitosamente las actividades académicas orientadas a la producción de información o evidencias. 
En cuanto a la dimensión input, la promedio general dimensión es de 1,89 puntos que equivale a $63,0 \%$, es decir, un nivel medio de la dimensión, con lo que se puede establecer que los estudiantes presentan un nivel medio de confianza para poder llevar a cabo las actividades académicas de insumo para el aprendizaje.

Para la dimensión retroalimentación el valor medio resultó ser 1,87 puntos, equivalente a 62,33\%, que se interpreta como un nivel medio de confianza para poder llevar a cabo las actividades académicas de interacción o retroalimentación del aprendizaje.

En otro sentido y para determinar las variables socio demográficas y académicas que constituyen el perfil del estudiante alrededor de su autoeficacia académica percibida, se utilizó el análisis de correlación con y sin causación (con valor de prueba de 0,050), empleándose así los estadísticos $t$ de Student, análisis de varianza y $r$ de Pearson según sea el caso. El rango para la media aritmética es de 0 a 3.

3.1 Resultados de diferencia de grupos y relación entre las variables sociodemográficas y académicas respecto al resultado global de autoeficacia académica percibida y sus dimensiones

El análisis de las variables sociales, demográficas y académicas que por su naturaleza se abordan a través de la prueba t de Student (con valor de prueba de 0,050) en relación con el resultado global de autoeficacia académica percibida, se muestra en la Tabla 2.

Tabla 2.

Variables sociales, demográficas y académicas en relación con el valor global de autoeficacia académica percibida

\begin{tabular}{|c|c|c|c|c|c|}
\hline \multirow{3}{*}{ Variable } & \multicolumn{5}{|c|}{ Autoeficacia académica percibida (global) } \\
\hline & \multirow{2}{*}{ Sig. } & \multicolumn{2}{|c|}{ Sí } & \multicolumn{2}{|c|}{ No } \\
\hline & & $\mu$ & $\sigma$ & $\mu$ & $\sigma$ \\
\hline Contar con algún tipo de beca & .033 & 1.95 & .044 & 1.81 & .048 \\
\hline Carrera de agrado (gusto por la carrera) & .000 & 1.95 & .034 & 1.59 & .092 \\
\hline Dificultades económicas para mantenerse en los estudios & .043 & 1.84 & .039 & 1.97 & .055 \\
\hline Deseos por estudiar algún posgrado & .000 & 1.99 & .039 & 1.66 & .050 \\
\hline Contar con profesionistas familiares & .249 & 1.94 & .051 & 1.86 & .042 \\
\hline Trabajar a la vez de estudiar & .849 & 1.91 & .096 & 1.89 & .035 \\
\hline $\begin{array}{l}\text { Vivir con sus padres en la localidad donde se ubica la } \\
\text { institución }\end{array}$ & .094 & 1.97 & .052 & 1.85 & .042 \\
\hline
\end{tabular}

Como puede observarse, las variables contar con algún tipo de beca, carrera de agrado, dificultades económicas para mantenerse en los estudios, y deseos por estudiar algún posgrado una vez que concluyan su licenciatura, muestran una diferencia estadísticamente significativa en quienes enuncian una respuesta afirmativa respecto a quienes no.

Esto quiere decir que quienes cuentan con algún tipo de beca expresan una autoeficacia académica mayor ( $\mu=1,95, \sigma=0,044)$ que quienes no cuentan con ella $(\mu=1,81, \sigma=0,048)$. A nivel dimensional, la diferencia se observa de manera específica en la dimensión de retroalimentación (significancia $=0,022$ ).

Quienes muestran agrado (gusto) por la carrera que estudian ( $\mu=1,95, \sigma=0,034)$ muestran una autoeficacia mayor a quienes no $(\mu=1,59, \sigma=0,092)$. A nivel intravariable, en las tres dimensiones existe estadísticamente diferencia significativa respecto a esta variable académica, teniendo la dimensión output una significación de 0,001, la dimensión input una significación de 0,001 y la dimensión retroalimentación una significación de 0,000. 


\section{Luis Fernando Hernández Jácquez}

Por su parte, la autoeficacia académica es mayor en quienes no muestran dificultades académicas para mantenerse en sus estudios $(\mu=1,84, \sigma=0,039)$. En este sentido la dimensión de autoeficacia output, es la única que presenta una diferencia significativa estadísticamente, respecto a esta variable (significancia $=0,003$ ).

Además, en todas las dimensiones de autoeficacia y en el resultado global se tiene una diferencia estadística en quienes tienen deseos de estudiar algún posgrado una vez que concluyan su licenciatura $(\mu=1,99, \sigma=0,039)$ respecto a quienes no $(\mu=1,66, \sigma=0,050)$, a saber: output $=0,005$, input $=0,000$ y retroalimentación $=0,000$.

En las variables contar con profesionistas familiares, trabajar a la vez de estudiar, vivir con sus padres en la localidad donde se ubica la institución, tipo de institución de procedencia (pública o privada); género (masculino o femenino) y estado civil (soltero o casado), no se puede establecer una diferenciación estadísticamente significativa respecto a la autoeficacia académica percibida (de manera global): $0,249,0,849,0,094,0,637,0,324$ y 0,165 , respectivamente.

Las variables tipo de bachillerato de procedencia y tipo de comunidad de procedencia en relación con la autoeficacia y sus dimensiones se abordaron mediante un análisis de varianza (ANOVA), el cual arroja que no puede establecerse una diferencia estadísticamente significativa entre estas variables, lo que se muestra en la Tabla 3.

Tabla 3.

Significancia estadística mediante ANOVA para las variables tipo de bachillerato de procedencia y tipo de comunidad de procedencia, respecto a la autoeficacia académica y sus dimensiones.

\begin{tabular}{lcc}
\hline Autoeficacia académica y sus dimensiones & \multicolumn{2}{c}{$\begin{array}{c}\text { Significancia estadística } \\
\text { Tipo de comunidad de } \\
\text { procedencia }\end{array}$} \\
\hline Output & Tipo de bachillerato de procedencia & .456 \\
Input & .671 & .627 \\
Retroalimentación & .880 & .119 \\
Autoeficacia académica (global) & .507 & .353 \\
\hline
\end{tabular}

Para finalizar, en la Tabla 4 se muestra el análisis correlacional para las variables que por su naturaleza deben abordarse a través del estadístico $r$ de Pearson, respecto a su asociación con la autoeficacia académica y sus dimensiones.

Tabla 4.Estadístico de Pearson para las variables señaladas respecto a la autoeficacia académica y sus dimensiones.

\begin{tabular}{|c|c|c|c|c|}
\hline \multirow[b]{2}{*}{ Variable } & \multicolumn{4}{|c|}{ Estadístico Pearson } \\
\hline & Dimensión output & $\begin{array}{l}\text { Dimensión } \\
\text { input }\end{array}$ & $\begin{array}{l}\text { Dimensión } \\
\text { retroalimentación }\end{array}$ & $\begin{array}{c}\text { Autoeficacia } \\
\text { académica } \\
\text { percibida }\end{array}$ \\
\hline $\begin{array}{l}\text { Cantidad de asignaturas no } \\
\text { aprobadas en el semestre } \\
\text { anterior }\end{array}$ & $-.210^{*}$ & $-.206^{*}$ & -.164 & $-.217^{*}$ \\
\hline Edad & -.105 & -.025 & .031 & .018 \\
\hline Promedio de bachillerato & $.201 * *$ & $.319 * *$ & $.220 * *$ & $.315^{* *}$ \\
\hline $\begin{array}{l}\text { Promedio del semestre } \\
\text { anterior }\end{array}$ & $.347 * *$ & $.415^{* *}$ & $.354^{* *}$ & $.445^{* *}$ \\
\hline $\begin{array}{l}\text { Puntaje obtenido en } \\
\text { examen de ingreso }\end{array}$ & $.214^{* *}$ & .014 & .033 & .075 \\
\hline
\end{tabular}


Como se observa, la relación que guarda el puntaje global de autoeficacia respecto a la cantidad de asignaturas que el estudiante no aprobó el semestre anterior al del estudio, es negativa a un nivel de significación de 0,05 ( $r=-0,217)$ lo que significa que si bien, es una relación débil, se puede establecer que conforme disminuye la cantidad de asignaturas no aprobadas, aumenta su percepción de autoeficacia académica, y viceversa. Lo mismo ocurre con la dimensión output ( $r=-$ $0,210)$ y la dimensión input $(r=-0,206)$.

El promedio de bachillerato guarda una relación positiva y moderada respecto a la autoeficacia (al nivel de significancia de 0,01 ) reflejándose tanto en el resultado global como en cada una de sus dimensiones (autoeficacia $=0,315$, output $=0,201$, input $=0,319$, retroalimentación $=0,220$ ), lo que significa que conforme aumenta el promedio que el estudiante haya obtenido en el bachillerato, aumenta su nivel de autoeficacia académica y viceversa.

Una relación también positiva pero con mayor fuerza, al nivel de significación de 0,01 se da entre la autoeficacia (y sus dimensiones) y el promedio obtenido por el estudiante en el semestre anterior (autoeficacia $=0,445$, input $=0,347$, output $=0,415$, retroalimentación=0,354), lo que significa que conforme aumenta (o disminuye) el promedio que el estudiante obtuvo en el semestre antecesor, aumenta (o disminuye) su autoeficacia percibida. Además de lo anterior, en el resto de variables no es posible establecer una relación estadística, a excepción de la dimensión output $(r=0,214)$ y el puntaje obtenido en el examen de ingreso a la institución, al nivel de significación de 0,01.

\section{DISCUSIÓN}

De manera global, el resultado de la autoeficacia académica percibida fue de 1,89 puntos, equivalentes a $63 \%$, lo que significa un nivel medio de autoeficacia percibida, y por lo cual es posible aceptar la primera hipótesis de investigación (H1 : El nivel de autoeficacia académica percibida en los estudiantes de una institución de educación superior del Estado de Durango, es medio).

En cuanto a la dimensión output, la media aritmética de 1,93 puntos significa que los estudiantes presentan un nivel medio de confianza para poder realizar exitosamente las actividades académicas orientadas a la producción de información o evidencias.

Para la dimensión input, el promedio general fue de 1,89 puntos, con lo que se puede establecer que los estudiantes presentan un nivel medio de confianza para poder llevar a cabo las actividades académicas de insumo para el aprendizaje; mientras que para la dimensión retroalimentación el valor medio resultó ser 1,87 puntos, que se interpreta como un nivel medio de confianza para poder llevar a cabo las actividades académicas de interacción o retroalimentación del aprendizaje.

Por otra parte, y a la luz del análisis de los resultados es posible determinar un perfil socioacadémico de los estudiantes que puede ser descrito a partir de su autoeficacia académica percibida, pero no un perfil que además de ello incluya variables demográficas por lo que rechaza H2, y se acepta H02: No es posible determinar un perfil sociodemográfico y académico de los estudiantes en cuestión, descrito a partir de su autoeficacia académica percibida.

A pesar de lo anterior, resulta muy útil poder determinar el perfil socio-académico asociado a la autoeficacia académica percibida, mismo que queda establecido de la siguiente manera: el nivel medio de autoeficacia académica percibida de los estudiantes está determinado por la seguridad de contar con algún tipo de beca, elgusto o agrado por la carrera que se estudia, el no tener dificultades 


\section{Luis Fernando Hernández Jácquez}

económicas para mantenerse en los estudios y por el deseo por estudiar algún posgrado una vez que se concluya la licenciatura.

Además, la cantidad de asignaturas que el estudiante no aprobó en el semestre antecesor, el promedio de bachillerato que se haya obtenido y el promedio del semestre anterior (en sus estudios de licenciatura) son variables que inciden directamente en la autoeficacia académica. Este último resultado, coincide en cierto sentido con lo reportado por Rosário (2012), quien concluyó que el rendimiento en matemáticas se puede predecir por la autoeficacia, y esta a su vez puede ser explicada por variables contextuales e incluso motivacionales, como las metas escolares, lo que conduciría al éxito escolar.

También coincide con Medrano (2011), quien estableció un modelo social cognitivo del rendimiento académico a partir de la autoeficacia. En otro sentido, de las variables descritas en el perfil, el contar con algún tipo de beca y el no tener dificultades económicas para estudiar, se pueden asociar a la fuente autoeficacia activación fisiológica ya que proveen la seguridad de que estos factores (beca y dificultades económicas) no serán una barrera que inhiba la autoeficacia.

El deseo por estudiar algún posgrado y el agrado por la carrera que estudian, son variables moduladoras de la autoeficacia intervinientes en la motivación, tal como fue señalado por Bandura (1997, citado por Chacón, 2006) al afirmar que las creencias de autoeficacia tienen una función fundamental en la autorregulación de la motivación. Las variables académicas como la cantidad de asignaturas no aprobadas, y los promedios del semestre anterior y de bachillerato, son experiencias directas (de dominio) como fuente de autoeficacia académica percibida de los estudiantes.

Este resultado, como todo perfil social y además académico, proporciona información que puede ser utilizada como diagnóstico de poblaciones con características similares a la que se estudió, y con ello, la institución podrá diseñar políticas y estrategias tendientes al aumento de su autoeficacia académica percibida, y por ende al mejoramiento de su desempeño académico.

En cuanto a la población estudiada (actual), el conocer sus características representa un acercamiento a sus condiciones sociales y académicas, con lo que las estrategias deberán ser precisas para intervenir en los factores sociales y académicos dependientes de la institución y sin duda alguna, en su autoeficacia.

En cuanto al resto de las variables involucradas en el estudio, como el contar con profesionistas familiares, trabajar a la vez de estudiar, vivir con sus padres en la localidad donde se ubica la institución, tipo de institución de procedencia, género, estado civil, tipo de bachillerato de procedencia, tipo de comunidad de procedencia, edad y puntaje obtenido en el examen de ingreso, no forman parte del perfil sociodemográfico directo que está asociado a la autoeficacia académica percibida de los estudiantes.

En relación a asociar a la autoeficacia con diversas variables del entorno, Blanco et al. (2012) determinaron diferencias entre hombres y mujeres respecto a su percepción de autoeficacia, al igual que Rosado (2013) quien lo señaló con su población de niños y niñas estudiada. La no coincidencia de los resultados del presente estudio con los de Rosado (2013) pudiese explicarse 


\section{Luis Fernando Hernández Jácquez}

porla edad tan distinta entre los sujetos de estudio, sin embargo, la población con la que trabajaron Blanco et al. (2012) parecería ser un tanto similar a la involucrada aquí, con excepción del nivel económico que es más alto en aquella. Aun así, con los resultados reportados en ambas investigaciones es difícil aseverar una explicación al respecto.

Por último, para Galicia et al. (2013), la cohesión familiar fue un factor que se relacionó positivamente con la autoeficacia académica en estudiantes de educación secundaria, situación que no se presenta en la población estudiada (estudiantes de educación superior y con las preguntas relacionadas al entorno familiar), por lo que puede deducirse que esta cohesión es importante para sujetos adolescentes, pero no lo es así en poblaciones de mayor edad; sin embargo se sugiere seguir investigando en este sentido para corroborar esta deducción.

Nota de Autores

El presente artículo de investigación forma parte de la línea institucional de investigación "Autorreferencialidad" de la Universidad Pedagógica de Durango, México

\section{REFERENCIAS}

Alpuche, A. \& Vega, L. (2014). Predicción del comportamiento lector a partir de la autoeficacia. Revista Mexicana de Investigación Educativa, 19(60), 241- 266. Disponible en https://www.comie.org.mx/v1/revista/portal.php?idm=es\&sec=SC03\&\&sub=SBB\&criterio=ART60010

Aluicio, A. \& Revellino, M. (2011). Relación entre autoeficacia, autoestima, asertividad, y rendimiento académico, en estudiantes que ingresaron a terapia ocupacional, el año 2010. Revista Chilena de Terapia Ocupacional, 11(2), 3-17. DOI: 10.5354/0719-5346.2011.17775

Alves, E. (2013). Autoeficacia de estudiantes de enfermería portugueses. Expresiones de creencia de autoeficacia, resiliencia y atribuciones casuales. (Tesis doctoral). Universidad de Extremadura, España. Disponible en http://dehesa.unex.es/bitstream/handle/10662/532/TDUEX_2013_Fonseca_EP.pdf?sequence=1

Bandura, A. (1977). Social Learning Theory. EUA: Prentice Hall.

Barraza, A. (2010). Validación del Inventario de Expectativas de Autoeficacia Académica en tres muestras secuenciales e independientes. CPU, 10, 1-30. Disponible en http://revistas.uv.mx/index.php/cpue/article/view/65/881

Barraza, A., Martínez, J., Silva, J., Camargo, E. \& Antuna, R. (2012). Estresores académicos y género. Un estudio exploratorio de su relación en alumnos de licenciatura. Visión Educativa IUNAES, 5(12), 33-43. Disponible en http://iunaes.mx/wp-content/uploads/2013/04/12-Marzo-de-2012.pdf

Blanco, A. (2010). Creencias de autoeficacia de estudiantes universitarios: un estudio empírico sobre la especificidad del constructo. Revista Electrónica de Investigación y Evaluación Educativa (RELIEVE), 16(1), 1-28. Disponible en https://www.uv.es/RELIEVE/v16n1/RELIEVEv16n1_2.pdf 


\section{Luis Fernando Hernández Jácquez}

Blanco, H., Ornelas, M., Aguirre, J. \& Gueda, J. (2012). Autoeficacia percibida en conductas académicas: diferencias entre hombres y mujeres. Revista Mexicana de Investigación Educativa, 17(53), 557-571. Disponible en https://www.comie.org.mx/v1/revista/portal.php?idm=es\&sec=SC03\&\&sub=SBB\&criterio=ART53010

Camposeco, F. (2012). La autoeficacia como variable en la motivación intrínseca y extrínseca en matemáticas a través de un criterio étnico. (Tesis doctoral). Universidad Complutense de Madrid, Madrid, España. Disponible en http://eprints.ucm.es/16670/1/T34002.pdf

Catzin, M., Esquivel, L. \& Canto, J. (2011). Perfil de estudiantes de preparatoria con riesgo de fracaso escolar. Educación y Ciencia, 10(34), 21-34. Disponible en http://www.educacionyciencia.org/index.php/educacionyciencia/article/view/257/pdf

Chacón, C. (2006). Las creencias de autoeficacia: un aporte para la formación del docente de inglés. Acción Pedagógica, 15, 44-54. Disponible en http://www.saber.ula.ve/handle/123456789/17262

Domínguez, S. (2016). Afrontamiento ante la ansiedad pre-examen y autoeficacia académica en estudiantes de ciencias de la salud. Educación Médica. Disponible en https://ac.els-cdn.com/S1575181316301097/1-s2.0S1575181316301097-main.pdf?_tid=477e75db-9e82-454d-8b0f3652a3c9855f\&acdnat=1520868906_23f8f058e9e0219807e048afb671c071

Galicia, I., Sánchez, A. \& Robles, F. (2013). Autoeficacia en escolares adolescentes: su relación con la depresión, el rendimiento académico y las relaciones familiares. Anales de Psicología, 29(2), 491-500. DOI: http://dx.doi.org/10.6018/analesps.29.2.124691

García, J., Díaz, A., Torregrosa, M., Inglés, C., Lagos, N. \& Gonzálvez, C. (2016). Capacidad predictiva de la autoeficacia académica sobre las dimensiones del autoconcepto en una muestra de estudiantes chilenos. Estudios sobre Educación, 30, 31-50. DOI: https://doi.org/10.15581/004.30.31-50

García, J., Inglés, C., Vicent, M., Gonzálvez, M., Lagos, N. \& Pérez, A. (2016). Relación entre la autoeficacia y autoatribuciones académicas en estudiantes chilenos. Universitas Psychologica, 15(1), 79- 88. DOI: http://dx.doi.org/10.11144/Javeriana.upsy15-1.raaa

Gómez, V., Villegas, C., Barrea, F. \& Cruz, J. (2007). Factores predictores de bienestar subjetivo en una muestra colombiana. Revista Latinoamericana de Psicología, 39(2), 311-325. Disponible en http://www.scielo.org.co/pdf/rlps/v39n2/v39n2a08.pdf

Hernández, L. (2016a). Autorregulación del aprendizaje y sus constructos asociados. Estado del arte. En D. Gutiérrez Rico, Cognición y Aprendizaje. Líneas de investigación (pp. 43-84). México: Plaza y Valdés Editores.

Hernández, L. (2016b). The evolution of academic selfefficacy and academic stress in the university students in Mexico. American Journal of Educational Research, 4(7), 556-562. DOI: http://dx.doi.org/10.12691/education-4-7-8

Hernández, R., Fernández, C. \& Baptista, M. (2014). Metodología de la Investigación. México: McGraw Hill. Sexta edición.

Disponible

en

https://trabajosocialudocpno.files.wordpress.com/2017/07/metodologc3a3c2ada_de_la_investigacic3a3c2 b3n_-sampieri-_6ta_edicion1.pdf 


\section{Luis Fernando Hernández Jácquez}

Kyle, T., Hernández, A., Reigal, R. \& Morales, V. (2016). Efectos de la actividad física en el autoconcepto y la autoeficacia en preadolescentes. Retos: nuevas tendencias en educación física, deporte y recreación, 29, 61-65. Disponible en https://recyt.fecyt.es//index.php/retos/article/view/36873

Medrano, L. (2011). Modelo social cognitivo del rendimiento académico en ingresantes universitarios. La contribución de la autoeficacia social académica. Revista Tesis, 1, 87-106. Disponible en https://revistas.unc.edu.ar/index.php/tesis/article/view/4119/3941

Méndez, M., Peña, J. \& Inda, M. (2012). Creencias de autoeficacia y elección femenina de estudios científicotecnológicos. Una revisión teórica de su relación. Teoría de la Educación, 24(1), 81-104. Disponible en http://revistas.usal.es/index.php/1130-3743/article/view/10333/10770

Ornelas, M., Blanco, H., Gastélum, G. \& Chávez, A. (2012). Autoeficacia percibida en la conducta académica de estudiantes universitarias. Formación Universitaria, 5(2), 17-26. Disponible en https://scielo.conicyt.cl/scielo.php?script=sci_arttext\&pid=S071850062012000200003\&lng=es\&nrm=iso\&tln $\mathrm{g}=\mathrm{es}$

Peralbo, M., Sánchez, J. \& Simón, M. (1986). Motivación y aprendizaje escolar: una aproximación desde la Teoría de la Autoeficacia. Infancia y Aprendizaje, 35(36), 37-45. Disponible en https://www.tandfonline.com/doi/abs/10.1080/02103702.1986.10822127

Piemontesi, S. \& Heredia, D. (2011). Relaciones entre la ansiedad frente a los exámenes, estrategias de afrontamiento, autoeficacia para el aprendizaje autorregulado y rendimiento académico. Revista Tesis, 1, 74-88. Disponible en https://revistas.unc.edu.ar/index.php/tesis/article/view/4118/3940

Polo, J. D., Santiago, V., Navarro, M. C. \& Alí, A. (2016). Creencias irracionales, síndrome de Burnout y adicción al trabajo en las organizaciones. Psicogente, 19(35), 148-160. http://doi.org/10.17081/psico.19.35.1215

Rosado, C. (2013). Los intereses ocupacionales y la autoeficacia académica en niños y niñas de escuelas elementales ubicadas en San Juan, PR. Cuaderno de Investigación en la Educación, 28, 36-60. Disponible en http://cie.uprrp.edu/cuaderno/download/numero_28/v28-2013-02.pdf

Rosário, P. (2012). Predicción del rendimiento en matemáticas: efecto de variables personales, socioeducativas y del contexto escolar. Psicothema, 24(2), 289-295. Disponible en http://www.psicothema.es/pdf/4013.pdf

Salkind, N. (1999). Métodos de Investigación. México: Prentice Hall.

Sánchez, J. (2013). Búsqueda de ayuda académica, autoeficacia social académica y emociones de logro en clase en estudiantes universitarios. Revista Argentina de Ciencias del Comportamiento, 5(1), 35-41. Disponible en https://revistas.unc.edu.ar/index.php/racc/article/view/5158/5323

Schunk, D. (2012). Teorías del Aprendizaje. Una perspectiva educativa. México: Pearson Educación.

Spiendler, S., Carlotto, M., Ogliari, D., Giordani, K. (2015). Estressores ocupacionais em psicólogos clínicos brasileiros. Psicogente, 18(33), 104-116. http://doi.org/10.17081/psico.18.33.59

Tuckman, B. \& Monetti, D. (2011). Psicología Educativa. México: Cengage Learning.

Uribe, J., Riaño, M., Bonilla, N., Carrillo S., Hernández, Y. \& Bahamón, M. (2017). Percepción de autoeficacia vs. rechazo del uso del condón en las prácticas sexuales de mujeres y hombres jóvenes. Psicogente, 20(37), 25-35. http://doi.org/10.17081/psico.20.37.2416

Psicogente, 20 (38): 25.34. Julio-Diciembre, 2017. DOI http://doi.org/10.17081/psico.20.38.2543 


\section{Luis Fernando Hernández Jácquez}

Veliz, A. \& Apodaca, P. (2012). Niveles de autoconcepto, autoeficacia académica y bienestar psicológico en estudiantes universitarios de la ciudad de Temuco. Salud \& Sociedad, 3(2), 131-150. Doi: http://dx.doi.org/10.22199/S07187475.2012.0002.00002

Está obra está bajo: Creative commons attribution 4.0 international license. El beneficiario de la licencia tiene el derecho de copiar, distribuir, exhibir y representar la obra y hacer obras derivadas siempre y cuando reconozca y cite la obra de la forma especificada por el autor o el licenciante 